\title{
Galaxy Clusters at the Edge: Temperature, Entropy, and Gas Dynamics at the Virial Radius
}

\author{
Jack O. Burns ${ }^{1}$, Samuel W. Skillman ${ }^{1,2}$, Brian W. O'Shea ${ }^{3}$ \\ jack. burns@cu.edu
}

\begin{abstract}
Recently, Suzaku has produced temperature and entropy profiles, along with profiles of gas density, gas fraction, and mass, for multiple galaxy clusters out to $\sim r_{200}$ ( $\approx$ virial radius). In this paper, we compare these novel X-ray observations with results from N-body + hydrodynamic adaptive mesh refinement cosmological simulations using the Enzo code. There is excellent agreement in the temperature, density, and entropy profiles between a sample of 27 mostly substructure-free massive clusters in the simulated volume and the observed clusters. This supports our previous contention that clusters have universal outer temperature profiles. Furthermore, it appears that the simplest adiabatic gas physics used in these Enzo simulations is adequate to model the outer regions of these clusters without other mechanisms (e.g., non-gravitational heating, cooling, magnetic fields, or cosmic rays). However, the outskirts of these clusters are not in hydrostatic equilibrium. There is significant bulk flow and turbulence in the outer intracluster medium created by accretion from filaments. Thus, the gas is not fully supported by thermal pressure. The implications for mass estimation from X-ray data are discussed.
\end{abstract}

Keywords: cosmology: theory — hydrodynamics — methods: numerical — temperatures — entropy — synthetic observations

\footnotetext{
${ }^{1}$ Center for Astrophysics and Space Astronomy, Department of Astrophysical \& Planetary Science, University of Colorado, Boulder, CO 80309

${ }^{2}$ DOE Computational Science Graduate Fellow

${ }^{3}$ Department of Physics \& Astronomy and Lyman Briggs College, Michigan State University, East Lansing, MI, 48824
} 


\section{Introduction}

Galaxy clusters are unique and potentially powerful cosmological probes of the Universe. They are the largest gravitationally bound objects, having grown hierarchically within the large-scale cosmic web. As such, clusters are an important source of information about the components of the universe in which they formed and evolved (e.g., Voit 2005; Borgani \& Kravtsov 2009). Gravity drives structure formation within an expanding universe, with regions of density higher than the average becoming gravitationally bound and decoupling from the expansion. Clusters probe the high density tail of this cosmic density field. The number density of clusters is strongly dependent upon the specific cosmological model, especially when viewed as a function of redshift (e.g., Rosati et al. 2002). The mass function of clusters and its evolution are also sensitive to $\sigma_{8}$ (a parameter that quantifies the RMS density fluctuations on comoving scales of $8 \mathrm{~h}^{-1} \mathrm{Mpc}$ ). The potential to use the cluster mass function to measure cosmological parameters is challenging observationally because mass is not a direct observational quantity. Rather, X-ray luminosity, X-ray temperature, thermal SZE (Sunyaev-Zeldovich Effect) integrated $Y\left(\propto \int \rho T d l\right)$, or weak lensing shear are the observables which must then be translated into an estimate of cluster mass.

Over the past decade, X-ray observations from ROSAT, Chandra, XMM-Newton, and, more recently $S u z a k u$, have begun to produce significant samples of clusters out to $z \approx 1.3$ (e.g., Allen et al. 2008). With these samples, it is becoming possible to distinguish between cosmological models using the evolution of the cluster mass function (e.g., Vikhlinin et al. 2009) and the cluster gas fraction (e.g., Rapetti et al. 2008). The primary limiting factor in such applications remains accurate conversion between X-ray observables such as X-ray luminosity and temperature to cluster mass. This is complicated by the nonlinear baryonic processes at the cores of clusters, including radiative cooling and non-gravitational heating from supernovae and active galactic nuclei (AGNs; e.g., Ruszkowski \& Begelman 2002; Heinz et al. 1998; Burns 1998) and possibly nonthermal processes involving cosmic rays (e.g. see Skillman et al. 2008) and magnetic fields (e.g., see Churazov et al. 2008; Xu et al. 2009). Other complications include possible bias and scatter in scaling relations (Motl et al. 2005; Nagai 2006), as well as errors created by assuming that cluster gas is in hydrostatic equilibrium (e.g., see Lau et al. 2009; Burns et al. 2008; Rasia et al. 2006; Nagai et al. 2007).

Because of the nonlinear nature of structure formation, the regions outside the cores do not necessarily behave in this same complex manner (e.g., Roncarelli et al. 2006). The peripheries of clusters have lower gas and galaxy densities, and long cooling times comparable to the Hubble time, thus potentially making the thermodynamics simpler than in the cores. At the same time, the outer regions of clusters are closer to the sources of accretion from filaments and, therefore, the gas dynamics may be more complex. 
The intracluster X-ray gas in galaxy clusters is often assumed to be in hydrostatic equilibrium which relates the gravitational potential $(\Phi)$ to the gas pressure $(P)$ and the gas density $(\rho)$ such that

$$
\nabla \Phi=-\frac{\nabla P}{\rho}
$$

Applying Gauss's Law to the gravitational potential in the above, the cumulative mass is

$$
M(<r)=\frac{1}{4 \pi G} \int-\frac{\nabla P}{\rho} d A
$$

where G is Newton's gravitational constant and the integral is over a spherical surface area with radius $r$. If the cluster is further assumed to be spherically symmetric and the pressure arises only from thermal motions $\left(P=\rho k T /\left[\mu m_{p}\right]\right)$, then

$$
M(<r)=-\frac{r^{2} k}{\rho G \mu m_{p}}\left[T \frac{d \rho}{d r}+\rho \frac{d T}{d r}\right] .
$$

As seen in Equation (3), we need to measure both the gas densities and temperatures, along with their gradients, to calculate the hydrostatic equilibrium masses. Gas density profiles can be accurately determined from the X-ray surface brightness, $S_{X}$, since $S_{X} \propto \rho^{2}$ with only a weak dependence on temperature.

Temperature profiles, on the other hand, are more challenging since they require spatiallyresolved X-ray spectroscopic observations acquired from relatively long integrations. This has been difficult, particularly to measure cluster temperatures beyond $\sim 0.5 r_{200} \sqrt{4}$. Because of the low and stable particle background levels at its orbit, Suzaku has begun to change the landscape by producing X-ray temperature profiles out to $\approx r_{200}$ for a small number of clusters including PKS 0745-171 (George et al.|2009), Abell 1795 (Bautz et al. 2009), Abell 399/401 (Fujita et al. 2008), Abell 2204 (Reiprich et al. 2009), and Abell 1413 (Hoshino et al. 2010). In each cluster, the temperature is observed to decline by a factor of $\approx 3$ from the peak near the cluster core to regions at $\approx r_{200}$.

Using the Eulerian adaptive mesh refinement cosmology code Enzo for a $\Lambda$ CDM universe, our group first proposed a universal temperature profile for galaxy clusters (Loken et al. 2002). This average profile was well-fit by a power-law out to the virial radius. This profile agreed well with the X-ray data available at that time for nearby clusters from $B e p$ poSAX (De Grandi \& Molendi 2002). Subsequent Chandra observations also appear consistent with such a universal temperature profile beyond the dense central cores for galaxy

\footnotetext{
${ }^{4} r_{200}$ is the radius at which the density is 200 times critical and $\approx$ virial radius for a flat $\Lambda$ CDM Universe (Bryan \& Norman 1998)
} 
clusters (e.g., Vikhlinin et al. 2006). However, these observed X-ray temperature profiles extended out only to $\approx 0.5 r_{200}$ so it was unclear if the universal temperature profiles for numerical clusters are in agreement with the outer profiles for real clusters.

In this paper, we compare the temperature, density, and entropy profiles for a new sample of numerical clusters generated using the Enzo cosmology code with new observations from Suzaku. We ask the question: Do real clusters follow a universal temperature profile out to the virial radius, as is predicted by numerical simulations with simple adiabatic gas physics? We also explore the implications of this particular form of the universal temperature profile for hydrostatic equilibrium, intracluster gas dynamics generated by on-going accretion in the outer periphery of clusters, and cluster mass estimation.

In Section 2, we describe the Enzo cosmology code and the numerical simulations used for the comparison with X-ray observations. In Section 3, we compare gas densities, temperatures, and entropy profiles between our numerical simulations and observed clusters. Then, in Section 4, we explore the implications of the form of the observed and simulated cluster temperature profiles on hydrostatic equilibrium and cluster mass determinations. We end with a summary and conclusions in Section 5.

\section{Simulations}

We use the Enzo adaptive mesh refinement (AMR) N-body + hydrodynamics cosmology code (Bryan \& Norman 1997a, b; Norman \& Bryan 1999; O'Shea et al. 2004; O'Shea et al. 2005) to simulate a comoving volume of $\left(128 h^{-1} \mathrm{Mpc}\right)^{3}$ with $256^{3}$ root-grid cells and up to 5 levels of additional refinement. This simulation utilizes the ZEUS finite-difference method (Stone \& Norman 1992a, b). The AMR is controlled by refining any region that is overdense by a factor of 8 in either the dark matter or gas density. The peak resolution is $15.6 \mathrm{~h}^{-1} \mathrm{kpc}$ (comoving). The initial conditions are generated from an Eisenstein \& $\mathrm{Hu}$ (1999) power spectrum with a primordial spectral index $n_{s}=0.97$. We use the cosmological parameters $\left(h, \Omega_{M}, \Omega_{B}, \Omega_{C D M}, \Omega_{\Lambda}, \sigma_{8}\right)=(0.704,0.268,0.0441,0.2239,0.732,0.9)$, with $h=H_{0} /\left(100 \mathrm{~km} \mathrm{~s}^{-1} \mathrm{Mpc}^{-1}\right)$. The simulation was initialized at $z=99$ and run until $z=0$. The dark matter mass resolution is $3.12 \times 10^{9} h^{-1} M_{\odot}$. For a further exposition of Enzo and its use in studying the statistical properties of clusters, see Skillman et al. (2008).

For all the analyses of the simulations and observations throughout this paper, we will assume $H_{0}=70 \mathrm{~km} / \mathrm{sec} / \mathrm{Mpc}$ and the other cosmological parameters given above. 
Table 1: Comparison of Properties of Simulated with Observed Clusters

\begin{tabular}{|l|l|l|l|}
\hline \hline Cluster & $z$ & $M_{200}\left(\mathrm{M}_{\odot}\right)$ & $r_{200}(\mathrm{Mpc})$ \\
\hline PKS 0745-191 & 0.103 & $6.4 \times 10^{14}$ & 1.72 \\
\hline Abell 1795 & 0.063 & $8.6 \times 10^{14}$ & 1.9 \\
\hline Simulated Clusters & 0.05 & $1.7-10 \times 10^{14}$ & $1.6-2.8$ \\
\hline \hline
\end{tabular}

\section{Comparison of Simulated and Observed Clusters}

We selected the 40 most massive clusters in our numerical volume at $z=0.05$ for comparison to the Suzaku observations because these clusters best match those observed (Table 11). This sample was then edited by examining the 3D distribution of the dark matter. Any cluster with a secondary dark matter density peak located outside of $0.1 r_{200}$ was marked as "disturbed". Upon visual inspection, these clusters were confirmed to be ones with major substructure. This produced a "clean" sample of the 27 most massive clusters that are relatively substructure-free. However, a note of caution is warranted, as there is accretion of smaller halos (subclusters) present in nearly all clusters; such small halo accretion is better seen in temperature images where shocks from supersonically merging subclusters are more obvious (see Fig. 1).

A grid of images of gas densities, temperatures, and $0.5-12.0 \mathrm{keV}$ X-ray emission is portrayed in Figure 1, For each temperature and density projection, we show a corresponding X-ray image. The method of Smith et al. (2008) was used for the radiative cooling to calculate frequency-dependent emission using the emission function from the Cloudy code (Ferland et al. 1998). Given a temperature and density of the gas, and assuming a metallicity of 0.3 solar, this returns the total X-ray emissivity. The X-ray surface brightness is the integral along the line of sight of the X-ray emissivity. These images in Figure 1 show a good deal of non-circularly symmetric structures, often produced by accretion along filaments, as also appears to be seen in the clusters observed by Suzaku (e.g., George et al. 2009; ;autz et al. 2009). 


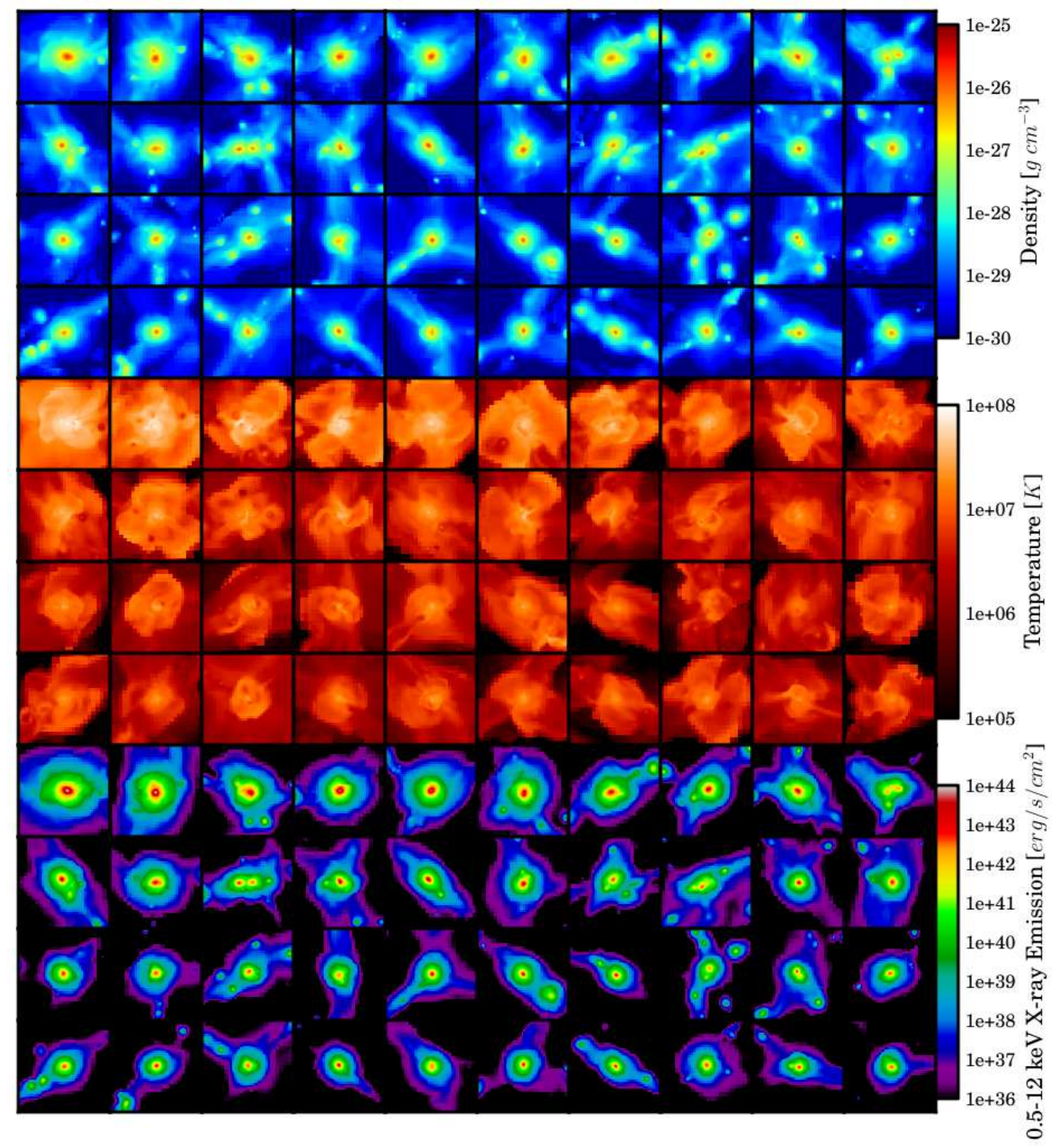

Fig. 1.- Grid of images of the 40 most massive clusters in the numerical sample selected from a cosmological volume at $z=0.05$. The images are ordered by $M_{200}$ from the upper left $\left(10^{15} \mathrm{M}_{\odot}\right)$ to the lower right $\left(1.7 \times 10^{14} \mathrm{M}_{\odot}\right)$ for each of the three panels of gas density (top), temperature (middle), and X-ray surface brightness (bottom). Field of view of each image is $8 h^{-1} \mathrm{Mpc}$. The range of properties for these clusters is listed in Table 1. This sample was reduced to 27 clusters for statistical analysis by eliminating obvious merger systems. 


\subsection{Analysis Pipeline}

\subsubsection{D Profiles}

For each cluster, we create spherically averaged radial profiles centered on the center of mass of the cluster. We track the following key fields: radius (in Mpc), overdensity of a sphere out to the current radius with respect to the background density $\left(\Omega_{m} \rho_{c r}\right)$, dark matter density, gas density, entropy, pressure, specific radial kinetic energy, specific kinetic energy, temperature, thermal energy, X-ray emission, total energy, and enclosed baryonic + dark matter mass.

An important point in our profiling procedure involves the various options for weighting the profiles. In this study, we use two different methods. First we can use the simple method of weighting by the mass of the cell. Therefore, regions in radial shells in high-density regions will be weighted higher than those in underdense regions. Second, we weight by the total $\mathrm{X}$-ray emission emanating from each cell. This biases towards overdense regions even more strongly since the X-ray emissivity scales roughly with $\rho^{2}$. In Figures 3 and 4 , we use Xray weighting to compare with X-ray observables. In Figure 5, we use mass weighting to investigate the physical causes of deviations from hydrostatic equilbrium.

\subsubsection{Profiles of 2D Projections}

Because observations yield inherently 2D images, it is important to compare projections of our clusters to the true observations. We do this by first creating halo projections of each cluster that encompass an $8 \mathrm{Mpc}$ cube around the individual cluster. For each of these projections, we again have a choice between weighting by the density or X-ray emission. Unless otherwise noted, we will use only the projections weighted by the X-ray emission. From each of the $2 \mathrm{D}$ projections, we then seek to create radial profiles. Here we give details of creating 2D profiles of X-ray weighted temperature, and use the same technique in all other quantities.

For each cluster, we first make a projection of the X-ray emissivity, $\epsilon_{X}$, which yields the X-ray surface brightness,

$$
S_{X, P r o j}(x, y)=\int \epsilon_{X}(x, y, z) d z .
$$

We then make a projection of the temperature, weighted by X-ray emissivity,

$$
T_{\text {Proj,X-Ray-weighted }}(x, y)=\frac{\int T(x, y, z) \epsilon_{X}(x, y, z) d z}{S_{X, P r o j}(x, y)} .
$$


From these quantities, we create the profiles of the $2 \mathrm{D}$ projections by weighting the temperature projection by the surface brightness,

$$
T_{2 D, X-\text { Ray-weighted }}(r)=\frac{\int T_{\text {Proj }, X-\text { rayweighted }}(x, y) S_{X, P r o j}(x, y) r d \theta}{\int S_{X, P r o j}(x, y) r d \theta},
$$

where $r$ and $\theta$ are the normal polar coordinates. This results in a correctly weighted radial profile of X-ray weighted temperature. Note that this is different than simply creating a 2D profile from the projection of X-ray weighted temperature. We can compare this final step to the equivalent step in the creation of the spherically averaged radial profile of the same quantity using normal spherical coordinates,

$$
T_{3 D, X-\text { Ray-weighted }}(r)=\frac{\iint T(x, y, z) \epsilon_{X}(x, y, z) r^{2} \sin \theta d \theta d \phi}{\iint \epsilon_{X}(x, y, z) r^{2} \sin \theta d \theta d \phi} .
$$

For an illustration of this pipeline, see Figure 2. 


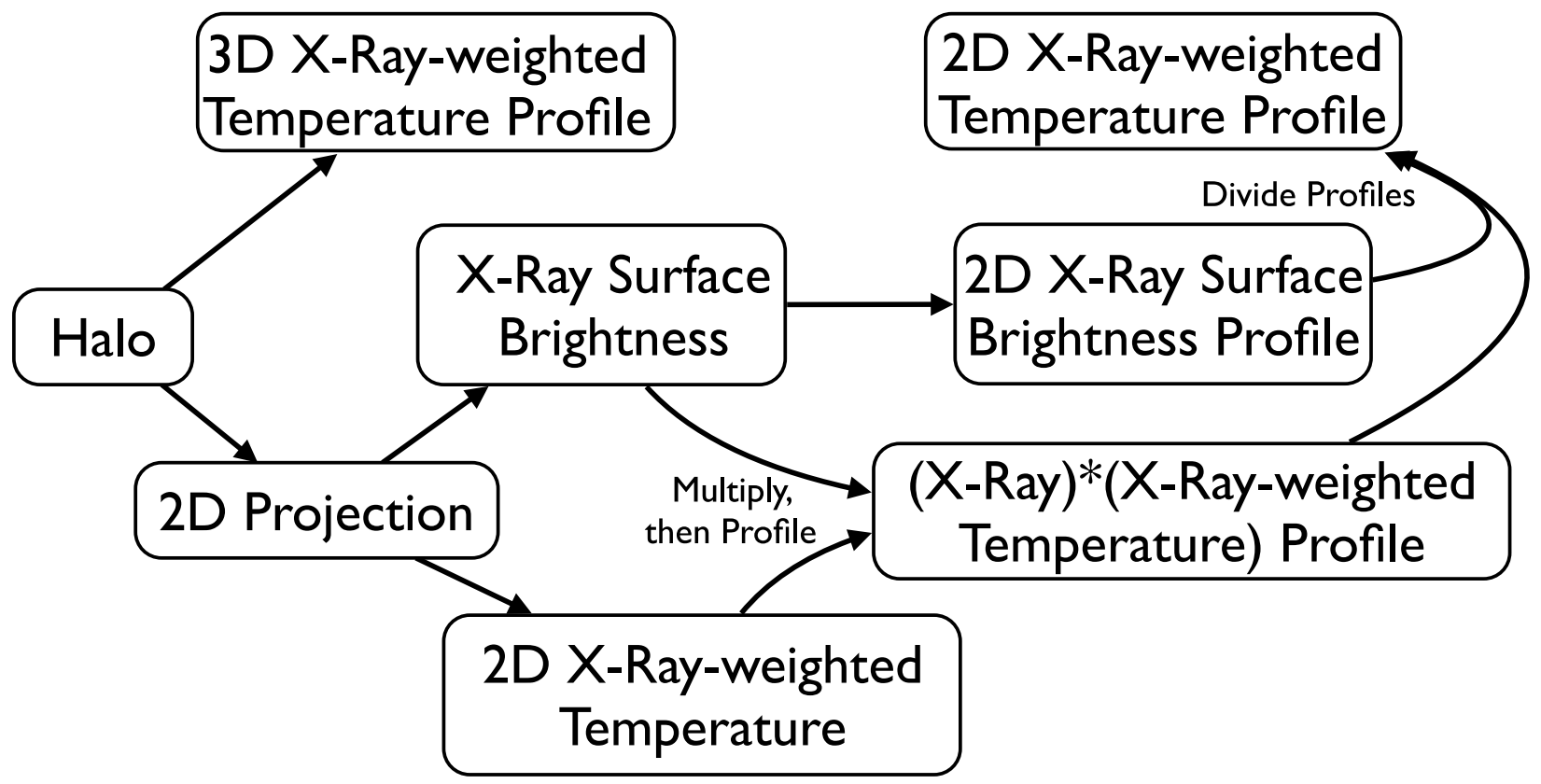

Fig. 2.- Analysis Pipeline to construct both a 2D and 3D X-ray weighted profile of a halo. 


\subsection{Results}

Applying the approach described in Section 3.1 to the analysis of the simulated clusters, we produced radial profiles of density, temperature, and entropy as shown in Fig. 3. Here, we define entropy as $K \equiv k T\left(\frac{\rho}{\mu m_{p}}\right)^{-2 / 3}$. The profiles were constructed by binning in circular annuli centered on the peak X-ray emission. The temperature profiles were each normalized to their X-ray weighted average values between 0.2 to $1.0 r_{200}$ so we could easily compare clusters of different masses. Such normalization is less effective for density and entropy which were, instead, normalized by values at $0.1 r_{200}$ and $0.3 r_{200}$, respectively. The shaded regions illustrate the standard deviations in quantities derived from the sample of numerical clusters. This scatter generally grows with radial distance, especially for the density and entropy, beyond $\approx 0.7 r_{200}$. This scatter reflects real variations from cluster to cluster in the outer regions produced by non-spherically symmetric accretion along filaments as mentioned above. The flat outer profile $\left(>0.5 r_{200}\right)$ seen for the average entropy of the numerical cluster sample in Figure 3 is, in part, due to the averaging of individual profiles, some with positive and some with negative slopes.

The average temperature profile shown in Figure 3 is well-fit by a function of the form

$$
\frac{T}{T_{\text {avg }}}=(1.74 \pm 0.03)\left[1+(0.64 \pm 0.10)\left(\frac{r}{r_{200}}\right)\right]^{-3.2 \pm 0.4}
$$

As we discuss in Section 4, the particular shape/slope of this power-law for the temperature profile has implications for understanding the gas dynamics and ICM pressure support in the outer regions of clusters.

In Fig. 3, we also compare the numerical profiles with observed ones for two nearby clusters (PKS 0745-191 and A1795) with recent Suzaku X-ray measurements that extend out to $\sim r_{200}$. A summary of the characteristics of these two observed clusters, along with the average properties of the numerical clusters, is given in Table 1. Both observed clusters have small cool cores. Since our simulations were purposely constructed with simple adiabatic gas physics, we did not expect to match the central regions of these clusters. Instead, we focus on the outer profiles, beyond $\gtrsim 0.5 r_{200}$. Other clusters recently observed by Suzaku have similar outer temperature profiles with a factor of $\approx 3$ decline in temperature between the core and $r_{200}$ (e.g., Reiprich et al. 2009; Hoshino et al. 2010). 

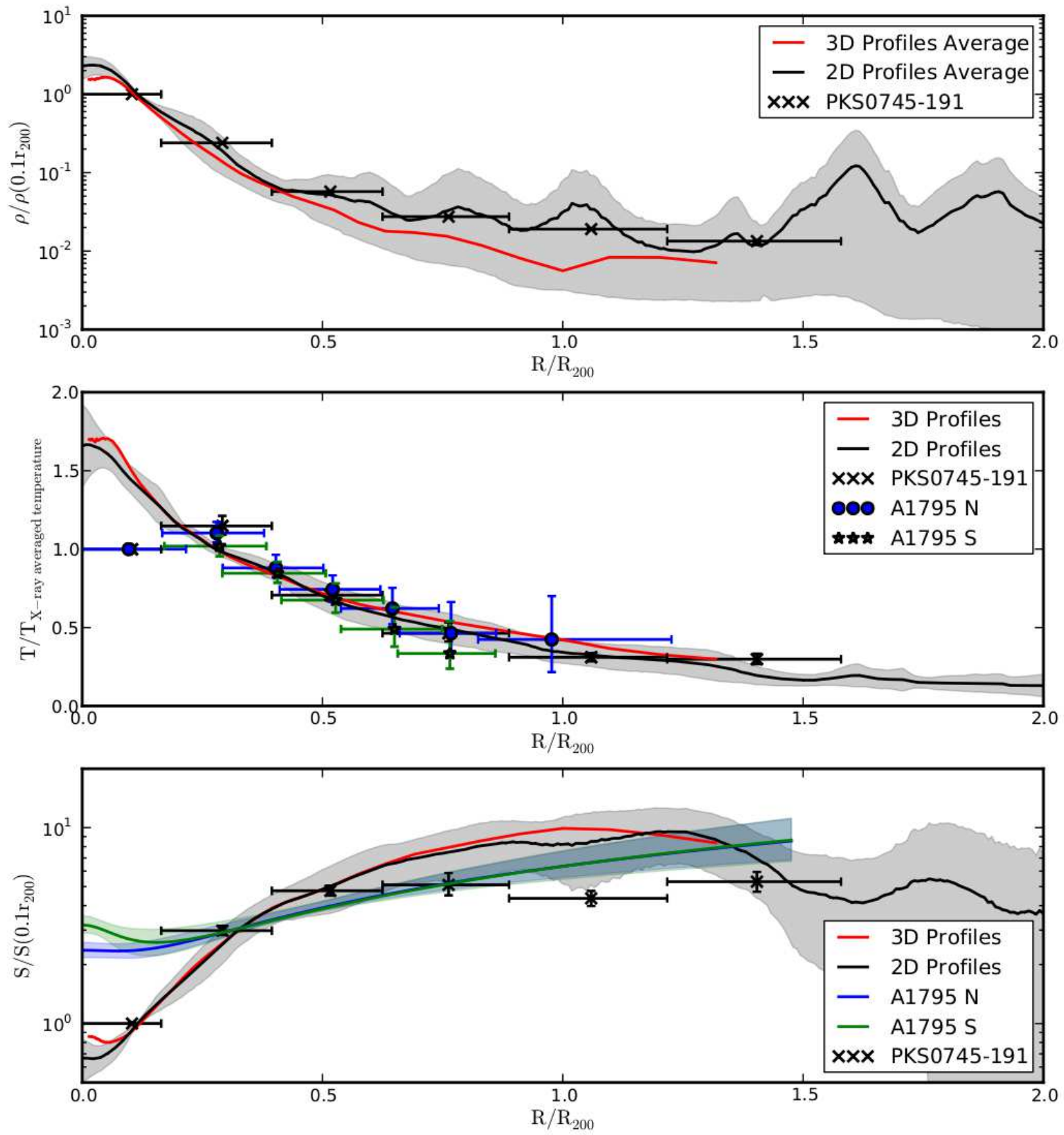

Fig. 3.- 3D and 2D X-ray weighted average profiles, as described in Section 3.1, of normalized density, temperature, and entropy for simulated clusters. The shaded regions are the standard deviations of each quantity produced from the scatter within the sample of numerical clusters. The Suzaku data points for PKS 0745-191 and A1795 (measured for two separate analysis regions, north (N) and south (S) of the core) are shown for comparison. The blue and green shaded regions in the bottom entropy profile are for the $\mathrm{N}$ and $\mathrm{S}$ analysis regions of A1795. The temperature is normalized by the average value between 0.2 to 1.0 $r_{200}$, whereas the density and entropy are normalized by values at $0.1 r_{200}$ and $0.3 r_{200}$, respectively. This difference in normalization produces a slight shift in the normalized entropy between simulations and PKS 0745-191. 
It is clear from this figure that the outer cluster radial distributions of density, temperature, and entropy for PKS 0745-191 and A1795 agree well with the simulated clusters. From Figure 3, we draw three conclusions about the regions outside the cluster cores. First, the observed clusters follow "universal" temperature, density, and entropy profiles as characterized by numerical simulations out to the outer bounds of the clusters $\left(r_{200}\right)$. Second, the simplest intracluster medium (ICM) gas physics with an adiabatic equation of state is sufficient to characterize the thermodynamic gas properties of the peripheries of these clusters. With the present Suzaku X-ray data, added gas physics such as cooling, non-gravitational heating (via e.q., low power radio or X-ray AGNs associated with non-central cluster galaxies (e.g., Hart et al. 2009)) or nonthermal pressure (due to ICM B-fields and cosmic rays (e.g., Skillman et al. 2008)) are not required, as also found by Roncarelli et al. (2006). Third, the nonspherical and dynamical gas/halo accretion environment present in the outer reaches of the numerical clusters is important in reproducing the observed properties, as is discussed in the next section.

\section{Discussion}

\subsection{Are Galaxy Clusters in Hydrostatic Equilibrium?}

What does the particular shape of the observed and numerical cluster temperature profiles imply about the dynamical state of the ICM? In particular, is this universal temperature profile consistent with the simplest form of hydrostatic equilibrium given in Equation (3), where the ICM pressure is strictly thermal?

In Figure 4, we compare the temperature profiles from the observed clusters and the simulations with that expected if the numerical clusters are in hydrostatic equilibrium. To calculate the hydrostatic temperature, we begin by making spherically-averaged 3D radial profiles of the dark matter density, weighted by the X-ray emission. From these radial profiles, we calculate the total enclosed mass as a function of radius. We then assume hydrostatic equilibrium to calculate the pressure derivative at each point,

$$
d P=-\frac{G M(r)\left(\epsilon \rho_{d m}\right)}{r^{2}} d r
$$

where $\epsilon=\rho_{\text {gas }} / \rho_{d m}$ is the ratio of gas to dark matter density and is assumed to be constant. We then integrate the pressure inwards, assuming $P=0$ at our outermost radial point. Finally, we calculate the temperature from

$$
T_{\text {hydro }}=\frac{\mu m_{p} P}{k \epsilon \rho_{d m}} .
$$


Because we normalize the temperature by the X-ray weighted average temperature between $0.2 r_{200}<r<r_{200}$, the absolute scaling of the pressure (and the value of $\epsilon$ ) does not affect the result. From the set of each individual profile, we then calculate the mean and standard deviation shown in Figure 4.

Using a $\chi^{2}$-test to compare the temperatures and their standard deviations for the simulated clusters in Figure 4, we find that the numerical 3D radial temperature profile and $T_{\text {hydro }}(r)$ have a $1.6 \%$ probability of being drawn from the same parent distribution. Although the scatter is large, the average hydrostatic temperature profile is $\approx 20 \%$ higher than the 3D numerical clusters or observed Suzaku clusters temperature profiles. This raises concerns about accurately calculating cluster masses using this method, especially for applications to precise cosmological parameter estimation.

In Figure 5, we investigate this issue further by plotting $\left(M_{\text {hydro }}(<r)-M_{\text {true }}(<\right.$ $r)) / M_{\text {true }}(<r)$, where $M_{\text {hydro }}(<r)$ is the mass within a sphere with radius $r$ calculated from hydrostatic equilibrium using Equation $(3)$ and $M_{\text {true }}(<r)$ is the dark matter mass. In this case, we use a mass weighting to probe the physical cause of the differences in mass. Previous numerical simulations have shown that there is a systematic bias (underestimate) of 5-15\% in the calculated mass of the central to mid regions of clusters $\left(r_{2500}-r_{500}\right)$ (Burns et al. 2008; Rasia et al. 2006; Lau et al. 2009) assuming hydrostatic equilibrium. Fig. 5 illustrates that this bias increases towards the edge of clusters. Beyond $\approx 0.8 r_{200}$, the average integrated mass is biased low by $\approx 15 \%$. Recent observational work by Mahdavi et al. (2008) supports this result in that they find hydrostatic cluster mass estimates are systematically low by 10-20\% compared to masses calculated via a weak lensing analysis out to $r_{500}$. Importantly, Figure 5 also illustrates the large scatter in the hydrostatic equilibrium calculation due to different dynamical states of individual clusters (even after we edited the sample for obvious mergers). At $0.8 r_{200}$, cluster hydrostatic masses range from $5 \%$ overestimates to $30 \%$ underestimates. 


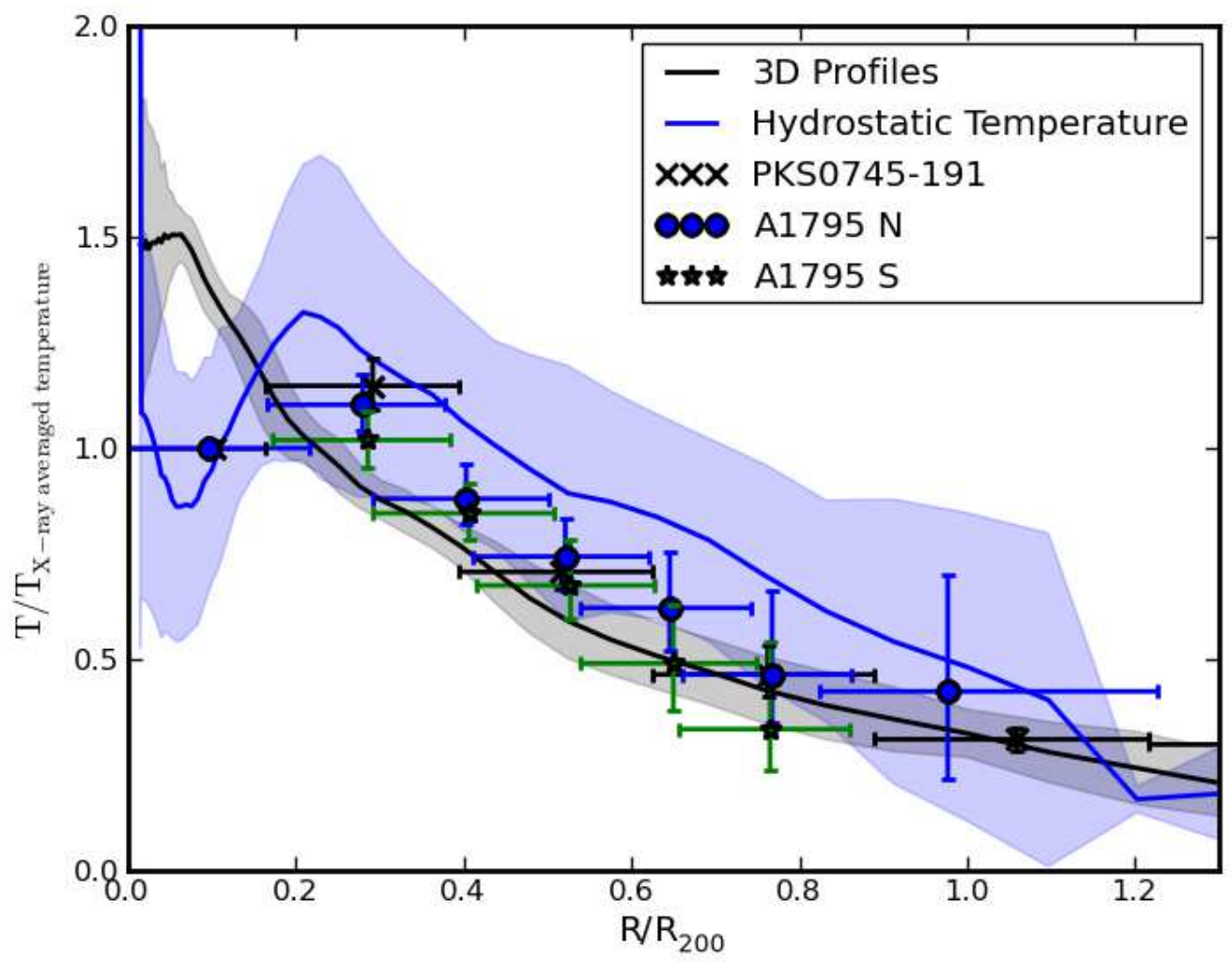

Fig. 4.- Comparison of numerical and observed cluster temperature profiles with that expected for hydrostatic equilibrium. The blue line and blue shading are the temperature distribution and standard deviations, respectively, expected if the gas in the dark matter potential wells of simulated halos is in hydrostatic equilibrium. The black line and grey shading are the X-ray weighted average temperature and standard deviations, respectively, for the numerical clusters. Suzaku data points are also shown for PKS 0745-191 and for two analysis regions (north and south) in A1795. 


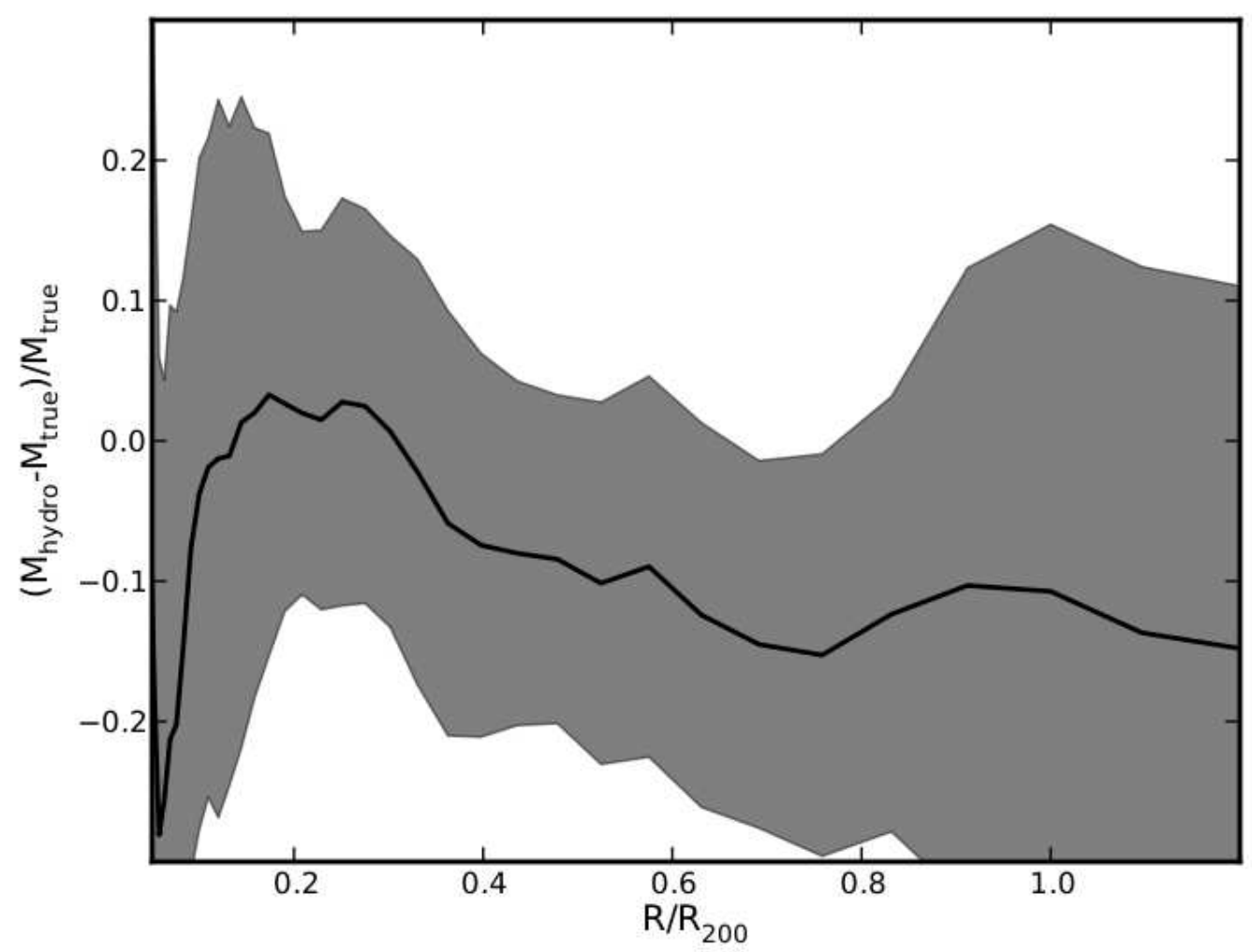

Fig. 5. - Radial profile of the fractional difference between cluster mass assuming hydrostatic equilibrium and the actual mass for the sample of numerical clusters. The shaded region is the standard deviation around the mean values. Note the increasing underestimate of mass with radial distance beyond $\approx 0.3 r_{200}$. 


\subsection{Gas Dynamics in the Outer Regions of Clusters}

Why does the intracluster medium of galaxy clusters not follow the simple prescription for hydrostatic equilibrium shown by Equation (3)? There are several obvious reasons which relate to the connection of clusters with the cosmic web and to their on-going evolution via accretion of gas and subclusters from filaments.

First, unlike the view of several decades ago, galaxy clusters are not simple spheres of gas and dark matter that are disconnected from their surroundings. Rather, they are closely tied to linear filaments within the large scale structure of the Universe. Gas, galaxies, and dark matter are funneled along these filaments into clusters, which typically lie at the intersections of the filaments. As a result, accretion onto clusters is complex and nonspherical. Thus, azimuthal variations in $\nabla P$ using Equation (2) need to be folded into the calculation of $M(<r)$.

Second, this accretion also leads to bulk and turbulent gas motions in the ICM. The bulk gas velocities are often hundreds to even thousands of $\mathrm{km} / \mathrm{sec}$ which may be detectable with upcoming high resolution X-ray calorimeter spectrometers (e.g., Astro-H XCS). One symptom of these bulk velocities and turbulent motions is the complex asymmetric temperature structures which are visible in simulated clusters as shown in Figures 1 and 6. The particular visualization in Figure 6 was chosen to display thin isocontours of temperature, highlighting the various phases of the intracluster gas. This temperature image illustrates the complex interplay between gas of different temperatures (and velocities) accreting from filaments at multiple angles and thermalizing via a web of shocks throughout the cluster.

Third, the kinetic gas motions are a significant fraction of the total energy density of the ICM, especially in the outer reaches of clusters. In Figure 7 , we plot the radial profiles of the ratios of the thermal $(n k T)$, radial kinetic $\left(\rho v_{r}^{2}\right)$, and turbulent kinetic energy (= difference between total and radial kinetic energy) densities to that of the total energy density of the gas in our numerical clusters. To calculate the kinetic energy density of the gas, we first find the center of mass velocity of the halo. We then compute the difference between a cell's velocity and this center of mass velocity. This then gives the kinetic motion relative to the halo. Both the kinetic energy and thermal energy are calculated using an X-ray emission weighting. Near the cluster centers $\left(\lesssim 0.2 r_{200}\right)$, the radial kinetic and turbulent kinetic energies contribute only $\approx 15 \%$ to the total energy of the ICM, consistent with mild biasing of the hydrostatic mass estimates in the central regions of clusters. However, at $r_{200}$, the radial kinetic energy equals the thermal energy. Furthermore, the turbulent energy density at the cluster edge is now $\approx 50 \%$ of the thermal energy and, thus, provides significant pressure support. So, it is not surprising that hydrostatic equilibrium increasingly fails as a valid assumption as we get closer to the cluster periphery. Turbulent gas motion is an important ingredient in 
the pressure support of galaxy clusters. For Equation (2) to result in an accurate estimate of cluster mass, it must contain a dynamical gas pressure term $\left(\sim \rho v^{2}\right)$ (see also Lau et al. 2009). 


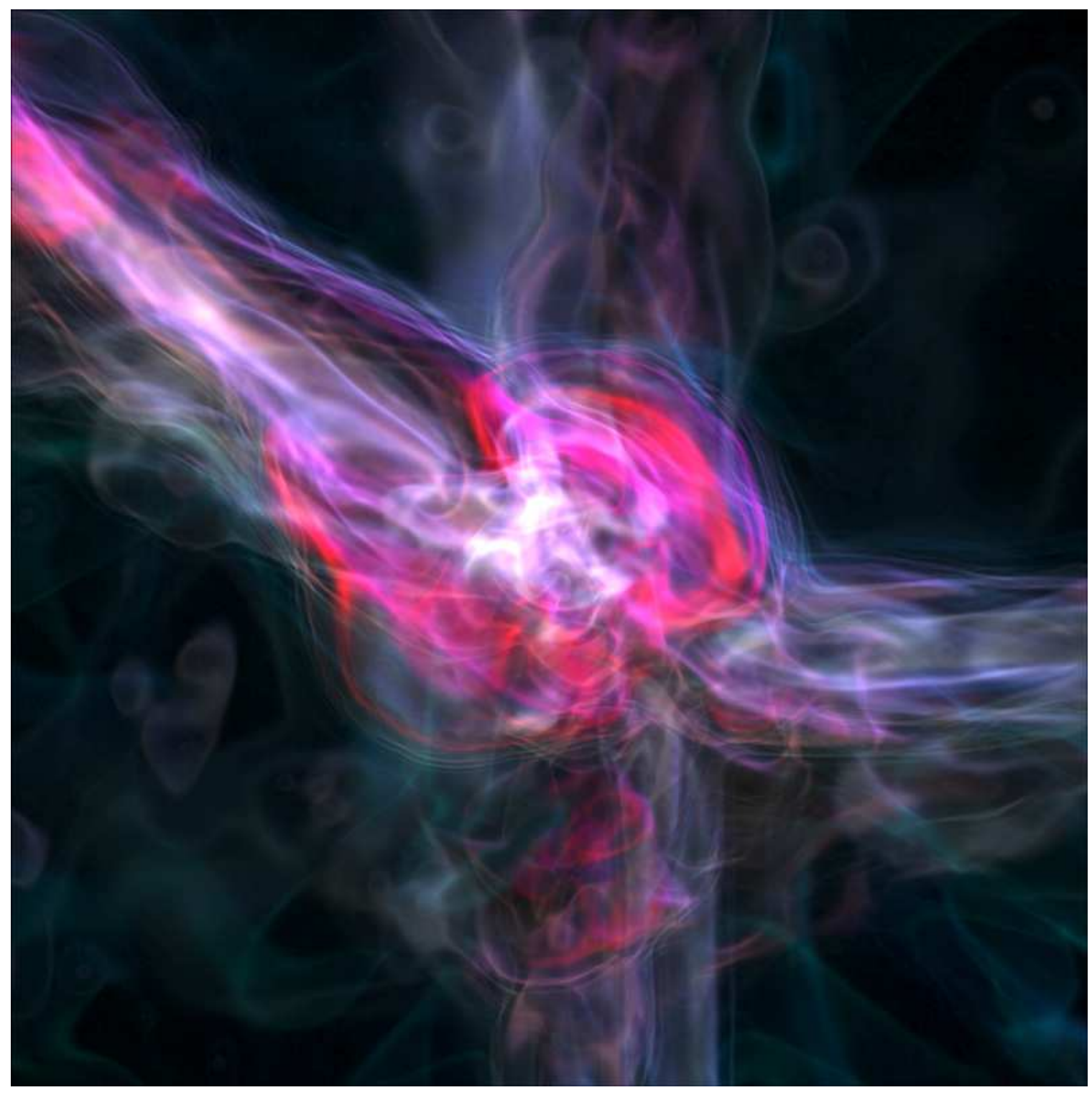

Fig. 6.- Isocontours of temperature for a numerical cluster. The field of view is 13.85 $h^{-1} \mathrm{Mpc}$. The colors correspond to the following temperatures: cyan $=10^{5} \mathrm{~K}$, magenta $=$ $3 \times 10^{5} \mathrm{~K}$, blue $=10^{6} \mathrm{~K}$, orange $=3 \times 10^{6} \mathrm{~K}$, red $=1.6 \times 10^{7} \mathrm{~K}$, and white $=5 \times 10^{7} \mathrm{~K}$. This new visualization was created using a ray casting module recently developed within $y t$. This image was made by first defining a red-green-blue-alpha transfer function based on any grid quantity. These emissivities are then integrated along a given ray from back to front. Note the complex interplay in temperature between the filaments, the cluster periphery, and cluster core. 


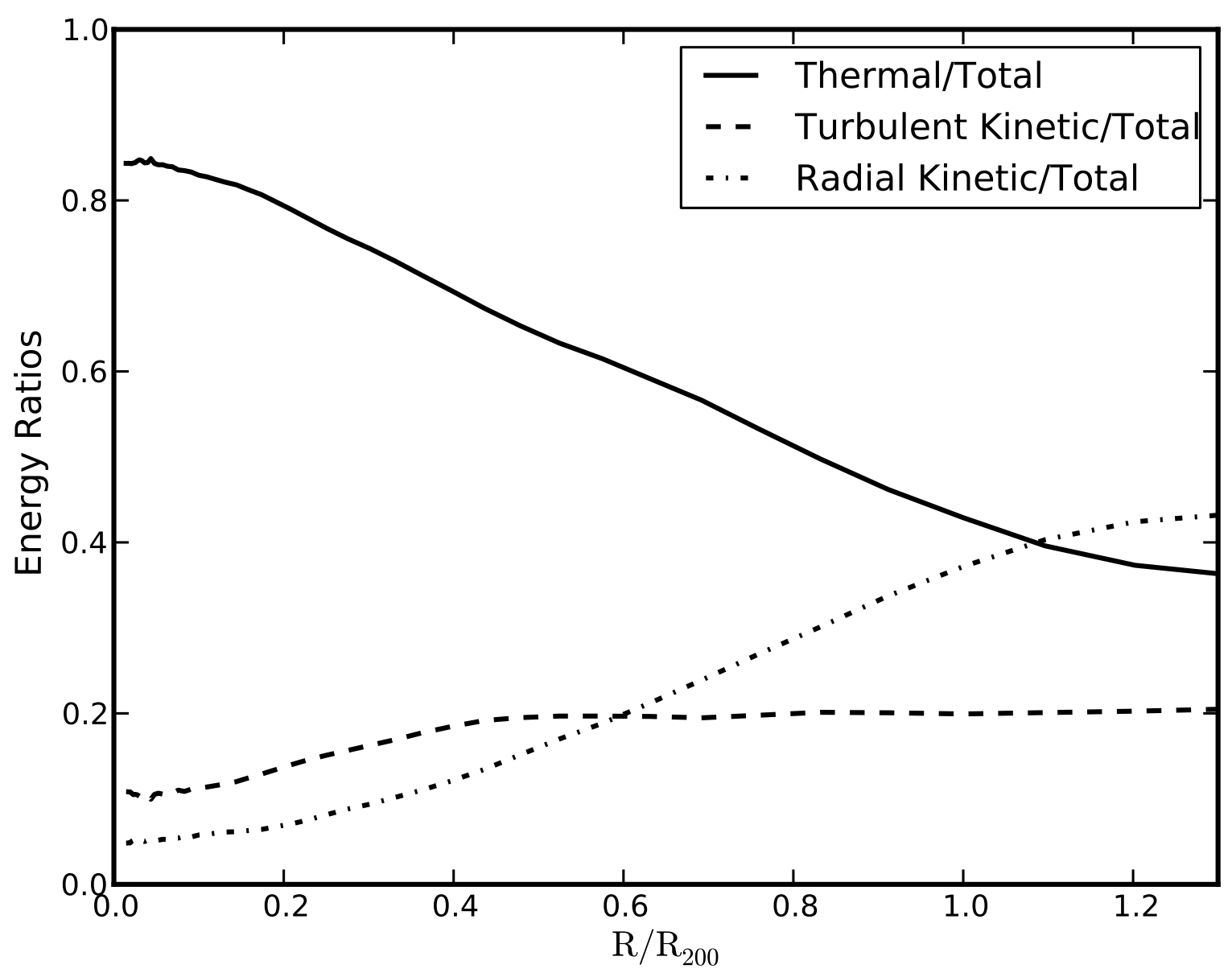

Fig. 7.- Energy density ratio profiles for the sample of numerical clusters. The thermal, radial kinetic, and turbulent kinetic energy densities are normalized to the total energy density within each shell. 


\section{Summary and Conclusions}

In comparing new Suzaku X-ray observations out to $\approx r_{200}$ for two rich galaxy clusters, PKS 0745-191 and A1795, with numerical clusters extracted from a large cosmological volume, we find that the observed clusters follow universal temperature, density, and entropy profiles. This excellent agreement between observations and simulations suggests that the simple adiabatic gas physics of the simulations appears to adequately define the thermodynamics outside the cores of these clusters. With the present Suzaku X-ray data, cooling, non-gravitational heating (via e.g., AGNs), magnetic fields, or cosmic ray pressure effects are not required.

These profiles, however, are not consistent with simple hydrostatic equilibrium in the outer cluster regions, suggesting that the ICM pressure support does not arise solely from the thermal energy density. Using cosmological numerical simulations, we show that nonspherical accretion from large-scale filaments and the resulting gas motions (radial and turbulent kinetic) have a comparable energy density to the thermal energy density near $r_{200}$. Assuming that thermal gas motions provides all the pressure support results in an increasingly inaccurate estimate of the total cluster mass with radial distance from cluster centers.

We thank Marc Bautz, Eric Miller, and Matt George for sharing their electronic files of the X-ray profiles for A1795 and PKS 0745-191, respectively. We also thank Eric Hallman, Britton Smith, Marc Bautz, Richard Mushotzsky, Eric Miller, and Megan Donahue for helpful conversations. Simulations were performed in part at the Los Alamos National Laboratory under the auspices of the Institutional Computing Program. J.O.B. acknowledges support from NASA ADP grant NNZ07AH53G and NSF grant AST-0807215. S.W.S. has also been supported by a DOE Computational Science Graduate Fellowship under grant number DEFG02-97ER25308. B.W.O. has been supported in part by a grant from the NASA ATFP

program (NNX09AD80G).Computations described in this work were performed using the Enzo code developed by the Laboratory for Computational Astrophysics at the University of California in San Diego (http://lca.ucsd.edu).

\section{REFERENCES}

Allen, S. W., Rapetti, D. A., Schmidt, R. W., Ebeling, H., Morris, R. G., \& Fabian, A. C. 2008, MNRAS, 383, 879

Bautz, M. W., Miller, E. D., Sanders, J. S., Arnaud, K. A., Mushotzky, R. F., Porter, F. S., Hayashida, K., Henry, J. P., Hughes, J. P., Kawaharada, M., Makashima, K., Sato, 
M., \& Tamura, T. 2009, PASJ, 61, 1117

Borgani, S. \& Kravtsov, A. 2009, ArXiv e-prints 0906.4370

Bryan, G. L. \& Norman, M. L. 1997a, 12th Kingston Meeting on Theoretical Astrophysics, proceedings of meeting held in Halifax; Nova Scotia; Canada October 17-19; 1996 (ASP Conference Series \# 123), 363

—. 1997b, Workshop on Structured Adaptive Mesh Refinement Grid Methods, ed. N. Chrisochoides (IMA Volumes in Mathematics No. 117), 433

Bryan, G. L. \& Norman, M. L. 1998, ApJ, 495, 80

Burns, J. O. 1998, Science, 280, 400

Burns, J. O., Hallman, E. J., Gantner, B., Motl, P. M., \& Norman, M. L. 2008, ApJ, 675, 1125

Churazov, E., Forman, W., Vikhlinin, A., Tremaine, S., Gerhard, O., \& Jones, C. 2008, MNRAS, 388, 1062

De Grandi, S. \& Molendi, S. 2002, ApJ, 567, 163

Eisenstein, D. J. \& Hu, W. 1999, ApJ, 511, 5

Ferland, G. J., Korista, K. T., Verner, D. A., Ferguson, J. W., Kingdon, J. B., \& Verner, E. M. 1998, PASP, 110, 761

Fujita, Y., Tawa, N., Hayashida, K., Takizawa, M., Matsumoto, H., Okabe, N., \& Reiprich, T. H. 2008, PASJ, 60, 343

George, M. R., Fabian, A. C., Sanders, J. S., Young, A. J., \& Russell, H. R. 2009, MNRAS, 395,657

Hart, Q. N., Stocke, J. T., \& Hallman, E. J. 2009, ApJ, 705, 854

Heinz, S., Reynolds, C. S., \& Begelman, M. C. 1998, ApJ, 501, 126

Hoshino, A., Henry, J. P., Sato, K., Akamatsu, H., Yokota, W., Sasaki, S., Ishisaki, Y., Ohashi, T., Bautz, M., Fukazawa, Y., Kawano, N., Furuzawa, A., Hayashida, K., Tawa, T., Hughes, J., Kokubun, M., \& Tamura, T. 2010, ArXiv e-prints 1001.5133

Lau, E. T., Kravtsov, A. V., \& Nagai, D. 2009, ApJ, 705, 1129 
Loken, C., Norman, M. L., Nelson, E., Burns, J., Bryan, G. L., \& Motl, P. 2002, ApJ, 579, 571

Mahdavi, A., Hoekstra, H., Babul, A., \& Henry, J. P. 2008, MNRAS, 384, 1567

Motl, P. M., Hallman, E. J., Burns, J. O., \& Norman, M. L. 2005, ApJ, 623, L63

Nagai, D. 2006, ApJ, 650, 538

Nagai, D., Vikhlinin, A., \& Kravtsov, A. V. 2007, ApJ, 655, 98

Norman, M. L. \& Bryan, G. L. 1999, Numerical Astrophysics : Proceedings of the International Conference on Numerical Astrophysics 1998 (NAP98), held at the National Olympic Memorial Youth Center, Tokyo, Japan, March 10-13, 1998., 19

O'Shea, B. W., Bryan, G. L., Bordner, J., Norman, M. L., Abel, T., Harkness, R., \& Kritsuk, A. 2004, eprint arXiv, Adaptive Mesh Refinement - Theory and Applications, 3044

O’Shea, B. W., Nagamine, K., Springel, V., Hernquist, L., \& Norman, M. L. 2005, ApJS, 160,1

Rapetti, D., Allen, S. W., \& Mantz, A. 2008, MNRAS, 388, 1265

Rasia, E., Ettori, S., Moscardini, L., Mazzotta, P., Borgani, S., Dolag, K., Tormen, G., Cheng, L. M., \& Diaferio, A. 2006, MNRAS, 369, 2013

Reiprich, T. H., Hudson, D. S., Zhang, Y., Sato, K., Ishisaki, Y., Hoshino, A., Ohashi, T., Ota, N., \& Fujita, Y. 2009, A\&A, 501, 899

Roncarelli, M., Ettori, S., Dolag, K., Moscardini, L., Borgani, S., \& Murante, G. 2006, MNRAS, 373, 1339

Rosati, P., Borgani, S., \& Norman, C. 2002, ARA\&A, 40, 539

Ruszkowski, M. \& Begelman, M. C. 2002, ApJ, 581, 223

Skillman, S. W., O'Shea, B. W., Hallman, E. J., Burns, J. O., \& Norman, M. L. 2008, ApJ, 689,1063

Smith, B., Sigurdsson, S., \& Abel, T. 2008, MNRAS, 385, 1443

Stone, J. M. \& Norman, M. L. 1992a, ApJS, 80, 753

-. 1992b, ApJS, 80, 791 
Vikhlinin, A., Kravtsov, A., Forman, W., Jones, C., Markevitch, M., Murray, S. S., \& Van Speybroeck, L. 2006, ApJ, 640, 691

Vikhlinin, A., Kravtsov, A. V., Burenin, R. A., Ebeling, H., Forman, W. R., Hornstrup, A., Jones, C., Murray, S. S., Nagai, D., Quintana, H., \& Voevodkin, A. 2009, ApJ, 692, 1060

Voit, G. M. 2005, Reviews of Modern Physics, 77, 207

Xu, H., Li, H., Collins, D. C., Li, S., \& Norman, M. L. 2009, ApJ, 698, L14 